\title{
Gestión de la felicidad y satisfacción del turista en Ecuador
}

\section{Happiness management and tourist satisfaction in Ecuador}

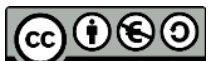

Gustavo Vladimir Paladines. ${ }^{1}$, Jenny Elizabeth Suárez Velasco. ${ }^{2}$ \& Segundo Fernando

\author{
Capa Paladines. ${ }^{3}$
}

Recibido: 12-07-2020 / Revisado: 10-08-2020 / Aceptado: 15-09-2020 / Publicado: 03-10-2020

\section{Resumen. \\ DOI: https://doi.org/10.33262/concienciadigital.v3i4.1432}

La gestión de la Felicidad o Hapiness Management es una práctica empresarial actual, que pone énfasis en la faceta humana del trabajador y en apoyar su bienestar; como resultado, la empresa se beneficia de contar con personal motivado, comprometido y leal; manifestándose una mayor calidad en su servicio, lo que deviene en un incremento de la satisfacción del cliente. El presente artículo aborda la gestión de la felicidad desde la posibilidad de su uso como recurso para mejorar la satisfacción del turista, y constituye un aporte al volumen actual de estudios referentes al Happiness Management. El artículo se elaboró mediante un método exploratorio, a partir de fuentes bibliográficas y documentales, tomando como referentes a diversas investigaciones en el ámbito de la felicidad en el contexto empresarial. Se observó que, tomando en cuenta los modelos de competitividad y las dimensiones de calidad para el turismo, la Gestión de la Felicidad es un recurso que podría tener un fuerte impacto en varios ámbitos, no solo en atención al cliente, sino en la capacidad de respuesta, en la confianza del cliente, en la reducción del ausentismo y abandono laboral, y en la imagen que el turista se forma de un servicio turístico, entre otros aspectos que reflejarían una satisfacción elevada en el turista.

Palabras claves: Turismo, Bienestar Social, Satisfacción En El Trabajo, Calidad En La Vida Laboral, Gestión.

\begin{abstract}
.
Happiness Management is a current business practice that emphasizes the human facet of the worker and it's care about improve his wellbeing; as result, the company will benefit from having motivated, involved and loyal staff; expressing a higher quality in its service;

${ }^{1}$ Universidad de las Fuerzas Armadas-ESPE, Escuela de Turismo, Sangolquí, Ecuador, gvpaladines@espe.edu.ec

${ }^{2}$ Universidad de las Fuerzas Armadas-ESPE, Escuela de Turismo, Sangolquí, Ecuador, jesuarez1@espe.edu.ec

${ }^{3}$ Universidad Internacional del Ecuador-UIDE-LOJA, Graduado de la Escuela de Gestión Turística y

Medioambiente, Loja-Ecuador, csegundofernando@yahoo.es
\end{abstract}


therefore, tourist satisfaction will grow. This paper approach to Happiness Management from the possibility of use as resource to improve tourist satisfaction, and it is a contribution to the low current volume from studies about Happiness Management. The study was conducted through an exploratory method, from bibliographical and documentary sources, considering as referents to taking as referring to various investigations in the field of happiness in the business context. Was observed that, considering the tourism competitiveness models, and tourism quality dimensions, Happiness Management is a resource that can have a strong impact in various areas. This impact is present not only in customer service, but in the capacity of response, the client's trust, in the reduction of job absenteeism and job abandonment, and, in the image that the tourist perceive from the tourist service, among others aspects that would reflect a high tourists satisfaction.

Keywords: Tourism, Social Welfare, Job Satisfaction, Quality In Working Live, Management.

\section{Introducción.}

El turismo es una actividad que debe tener a la calidad como criterio primario en todas las expresiones del servicio que dé como resultado la satisfacción del visitante. Griffiths, Johnson y Hartley (2007) establecen tres posturas respecto a la satisfacción. Como estado o respuesta mental (racional o emocional) a un estímulo; como la reacción corporal y/o mental hacia un servicio o producto; y, como la sensación del cliente de que un producto o servicio utilizado cumple o excede sus expectativas y requerimientos. Desde estas perspectivas, para el turista la experiencia en el destino visitado debe ser completamente satisfactoria, de otra manera sentirá que su decisión fue errónea y, con altas probabilidades, no recomendará o utilizará el mismo servicio en otra ocasión.

La satisfacción del turista se produce por la suma de distintos factores, y, acogiendo el modelo de calidad del servicio de Parasuraman, Zeithalm y Berry (1985), se identifican cinco dimensiones de calidad en el servicio: elementos tangibles, fiabilidad, capacidad de respuesta, seguridad y empatía. De estas dimensiones, a excepción de los elementos tangibles, todas dependen de forma directa 0 indirecta, del personal que brinda el servicio. Por esto, es concluyente que el factor humano es un elemento clave y determinante de la calidad del servicio prestado, y su desempeño y actitud dependen a su vez de la forma en que el personal se siente en su lugar de trabajo.

Con el pasar del tiempo han surgido diversas iniciativas encaminadas a evaluar, analizar y gestionar las condiciones de trabajo con la finalidad de impactar de forma positiva en el trabajador. Entre estas iniciativas se encuentra la ergonomía, el clima laboral, la gestión del estrés, el análisis de carga laboral, entre otras. No obstante, en los últimos años surgió el llamado happiness management como práctica que abarca de forma integral, la gestión de todos los aspectos que aseguren que el personal se sienta feliz de laborar en la organización.

Para poner en contexto el tema, la Organización Mundial del Turismo proyectó en el año 2011 su análisis Tourism Towards 2030: Global Overview un crecimiento de las llegadas de turistas internacionales a nivel mundial en un $+3,3 \%$ anual hasta el año 2030. Sin embargo, en la práctica 
las expectativas se superaron, con un $+7 \%$ y $+6 \%$ de crecimiento en llegadas de turistas internacionales para los años 2017 y 2018 respectivamente (World Tourism Organization, 2019). En Suramérica el crecimiento fue de $+7 \%$ en el año 2017 y $+3 \%$ en el 2018. En el caso de Ecuador, los terremotos acaecidos en abril del año 2016 debilitaron su papel en el turismo en comparación con otros países de la región, decreciendo en $-8,2 \%$, pero recuperándose para el año 2017 con un $13,4 \%$ que representó un total de1' 608,000 de llegadas de turistas internacionales. En comparación con la región su participación fue del 4,4\% (Organización Mundial del Turismo, 2018).

Esto muestra un aumento importante del turismo a nivel mundial, y una oportunidad de desarrollo para economías emergentes; que deben poder obrar de forma acertada para captar, mantener y elevar su participación dentro del mercado turístico mundial; logrando turistas satisfechos con su experiencia. En el enfoque de este artículo, se considera que la calidad de la atención y la actitud que el turista percibe en el personal de los servicios turísticos es un determinante de su satisfacción. En contexto, según el Ministerio de Turismo en Ecuador el sector turístico registró 463320 empleados para el cuarto trimestre del año 2018, una cifra significativa en comparación a los 2,4 millones de extranjeros que ingresaron al país ese mismo año, es decir, cerca de 1 trabajador por cada cinco turistas.

Respecto a la satisfacción del turista en Ecuador, no existen estudios a nivel nacional, sin embargo, se han encontrado diversas investigaciones que hacen referencia a este factor en destinos puntuales.

Cevallos (2013) evaluó la satisfacción de los turistas en su visita a las Islas Galápagos, y obtuvo como resultado un $10 \%$ que calificaron como "nivel de satisfacción regular" a los servicios de alimentación y de hospedaje.

Espín (2014) evaluó el nivel de satisfacción del turista, respecto de los servicios de alojamiento en Baños de Agua Santa, y encontró que los niveles de insatisfacción llegaron al 23\% en alojamiento, al $15 \%$ en restaurantes, y $27 \%$ en servicio a la habitación; con un $42 \%$ que no recomendaría el servicio a otras personas.

En el estudio de Varenius (2016) se evaluó la satisfacción de los turistas que visitan la Isla Santay en la ciudad de Guayaquil. El autor encontró que los niveles de satisfacción eran bajos en cerca del $10 \%$ de los visitantes, lo que implica una cifra reducida frente a un 50\% aproximadamente que se mostró muy satisfecho con diversos aspectos de su experiencia, tratándose de turismo de naturaleza.

Álava y Bastidas (2016) evaluaron la satisfacción de los turistas respecto a las agencias de viaje en Puerto Ayora, en las Islas Galápagos, y encontró que el 28\% de turistas no se sintió satisfecho con el desempeño de la agencia de viajes utilizada y el $24 \%$ valoró como "regular" el interés del empleado al momento de realizar preguntas sobre el servicio a adquirir, por lo que el $21 \%$ no se sintió satisfecho con el comportamiento del empleado.

De acuerdo con Carvache W., Torres y Carvache M. (2017) los turistas que han visitado Montañita, principalmente turistas jóvenes de entre 18 y 24 años, valoraron la satisfacción de la 
atención del personal en servicios hoteleros en 3,92 / 5 (78\%) y en servicios de restaurantes en 3,78/5 (siendo 5 la mayor satisfacción). En este punto la satisfacción en atención se alcanzó en un $78 \%$ y $76 \%$ de forma respectiva. Otro estudio similar realizado en General Playas Villamil, de la provincia del Guayas, Ecuador, elaborado por Carvache M., Carvache, W., Macas y Orden (2018) registraron la satisfacción del turista en dicha locación como satisfechos en el 45,2\% y muy satisfechos en el 36,4\%; dejando a un 18,4\% como indiferentes o insatisfechos,.

En los estudios encontrados entre el 2013 y 2018, se observa un decrecimiento de la satisfacción del turista al pasar los años, lo que también puede deberse a una disminución en las actividades e inversión del Estado mediante el Ministerio de Turismo, las cuales pasaron, de USD 94,1 millones en el año 2015 a USD 8,7 millones en el 2017 y a un presupuesto de USD 4,5 millones para el año 2019 (Encalada, 2019). Esta reducción tiene efectos directos como una menor inversión en publicidad y promoción turística del país, recortes en el presupuesto para el desarrollo de destinos turísticos, e incluso una disminución en actividades de orientación y capacitación turística para pequeños y medianos empresarios del país. Esta afectación en la cantidad de turistas, y a la vez, en la cantidad de inversión que el sector recibe, requiere que se incremente el aprovechamiento de los recursos y que se logren los mejores resultados.

En este análisis se vislumbra la posibilidad de que, mediante la aplicación del Happiness Management, los proveedores de servicios turísticos puedan mejorar la calidad percibida en el servicio por el turista, a la vez que se benefician de las ventajas que esta práctica tiene tanto dentro como fuera de la organización.

Los prestadores de servicios turísticos en el país no pueden depender de organismos de apoyo para que evalúan o establezcan parámetros que determinen sus niveles de calidad, sin embargo, en el caso de micro y pequeños empresarios, es común que no exista el conocimiento de prácticas administrativas o técnicas que apoyen el logro de la mejora continua, al tratarse, con frecuencia, de pobladores del destino turístico que complementan la oferta empresarial, con servicios de alimentación, transporte e incluso alojamiento. Sin embargo, ya sean proveedores independientes, o empresas legalmente constituidas, adquieren la responsabilidad de proveer un servicio de excelencia que sea compatible con la imagen turística que el país quiere proyectar. La gestión de la felicidad es una alternativa que, correctamente aplicada, se estima que tendría un impacto positivo en el desempeño del personal y en la forma en que se comporta con los turistas.

Para profundizar en el tema vale considerar el fundamento teórico de la gestión de la felicidad. El denominado Happiness Management es un concepto que tiene aún poca difusión, que propone una gestión del talento humano encaminada a desarrollar las condiciones de trabajo que aseguren la felicidad del trabajador. No obstante, el concepto ahonda en una mayor profundidad como sugieren Rai, Singhi y Gupta (2012), quienes plantean que los Sistemas de gestión utilizados hasta el momento, como la gestión por desempeño o por competencias, apuntan al rendimiento que tiene lugar en el trabajador como efecto de sus habilidades cognitivas o sus destrezas, siendo un concepto limitado pues socava otros aspectos importantes como espirituales, sociales y 
psicológicos. Así, la gestión de la felicidad se fundamenta en los planteamientos de Aristóteles, para quien la felicidad es el propósito más elevado de la vida.

Si se considera la pirámide de necesidades de Maslow, cuando las necesidades básicas (fisiológicas y de seguridad) están resueltas, la persona se hallará en búsqueda de satisfacer necesidades sociales o de pertenencia, y por último, aquellas de tipo interno o espiritual, nombradas por Maslow como necesidades de estima y autorealización (Turienzo, 2016). Sin embargo, en las organizaciones es habitual que solamente las necesidades básicas suelan asegurarse, mientras que las necesidades de pertenencia, menos aún las de autorrealización, pueden ser satisfechas, esto debido a que no se considera a estas como fundamentales para el desempeño del trabajador. En la postura del Hapiness Management estas necesidades toman mayor relevancia, pues el trabajador satisfecho se sentirá feliz, pero a su vez, un trabajador feliz sentirá que sus necesidades son menores. De modo que esta retroalimentación de ambos factores (satisfacción y felicidad), beneficia a la organización y al trabajador. Para Edwards (2009) este modelo de gestión surge como contraposición al miedo como forma de control y motivador que solía prevalecer en el sector industrial (miedo al despido por ejemplo), y que afectaba a la moral del empleado a largo plazo; y se centra en el bienestar subjetivo del individuo (Wesarat, Yazam, \& Abdul, 2015).

No obstante, en este punto es necesario diferenciar la satisfacción de la felicidad. De acuerdo con Ayu y Lyana (2018) no existe un consenso sobre lo que es la felicidad. Los conceptos abarcan posturas filosóficas o psicológicas, definiéndolo como la más importante emoción lograda mediante el bienestar o la satisfacción, o como el cumplimiento de todos los deseos a lo largo de un período de vida. Hartini, Khalil y Zakaria (2017) consideran felicidad como una emoción básica caracterizada por un estado emocional marcado por la sensación de bienestar y placer, junto con un sentimiento de éxito asociado con un claro entendimiento del mundo, mientras que la satisfacción es una autoevaluación de los sentimientos y actitudes sobre un punto particular en el tiempo, que puede ir de negativo a positivo. Para Xinggui, Yang, Ming y Zhanpeng (2018) la felicidad forma parte de la personalidad del ser humano, mientras la satisfacción es una reacción a situaciones externas.

Retomando el modelo de Maslow, la satisfacción de los diversos niveles de necesidades solo culminaría con el logro de necesidades de autorrealización (la punta de su pirámide). En teoría una persona se sentiría satisfecha, y por ende feliz, al tener todas sus necesidades satisfechas, pero el ser humano es un ser cambiante, inconforme, y que se siente motivado en la búsqueda más que en el logro. Dicho de otro modo, nunca existirá un estado de satisfacción perfecta, pues el ser humano, con frecuencia, anhelará alcanzar algo más o algo diferente a aquello que ya logró. La felicidad en cambio, puede tornarse en un estado emocional y mental más estable y constante.

En cuanto al ámbito turístico, los modelos de excelencia suelen ubicar al personal en una o dos de las dimensiones de calidad (generalmente en gestión de personal y capacidad de respuesta), es decir, que se concentran en los resultados del servicio. Desde una perspectiva de gestión de la felicidad, el impacto positivo producido por el personal que se siente feliz por su trabajo, incidiría en una mejora general de la actitud de trabajo, de servicio, tanto con el turista como con sus 
compañeros de trabajo. El turista que es testigo de un servicio en el cual el personal se muestra feliz, y consecuentemente amable y predispuesto a servir, se sentirá satisfecho y percibirá una calidad elevada en el servicio.

El propósito del estudio es establecer la relación empírica entre la gestión de la felicidad como estrategia empresarial y la satisfacción de los turistas. Como sugiere Aranda (2016) "entre más feliz es un empleado, mayor será su productividad". En el caso del sector turismo se esperaría que el personal que se encuentra más feliz en su puesto de trabajo, manifieste una actitud positiva y mayor predisposición para solventar las necesidades de los turistas.

De acuerdo con Romero, Castillo, y Ravina (2019) la felicidad, al igual que el desempeño de un trabajador, depende de su función en la organización (contribución), motivación (convicción), sentido de pertenencia (cultura), apego emocional a la organización (compromiso) y confianza en su potencial (capacidad). Bajo esta postura, el trabajador se siente feliz cuando considera que su rol en la organización es importante, y si es reconocido, se siente, además, motivado. Si la cultura interna le ayuda a desarrollar una sensación de pertenencia y afiliación emocional a la empresa, el trabajo ya no será únicamente una obligación, sino una responsabilidad que cumplirá motivado, lo cual eleva su confianza en sus capacidades. Sin embargo, lo relevante del concepto de felicidad es que, como se señaló previamente, es un estado emocional y mental, que se genera por estos factores y al mismo tiempo los fortalece.

La satisfacción es un factor que promueve la felicidad, pero, de manera dual, la felicidad apoya la satisfacción. Ambos elementos se desarrollan de manera casi paralela. La premisa del estudio propone que un trabajador feliz en el sector turístico se desempeñará mejor en sus funciones, se sentirá parte de la organización, compartirá sus logros, e incluso, trasmitirá esta actitud positiva a quienes brinde sus servicios. Por lo mismo, excederá las expectativas de los turistas desarrollando un sentimiento de satisfacción en estos por el servicio recibido, y contribuye también a que su experiencia les genere felicidad.

\section{Metodología.}

El presente artículo es una investigación empírica de carácter exploratorio, puesto que aborda la potencial relación entre la felicidad de los trabajadores y la satisfacción de los turistas a quienes sirven. Las fuentes utilizadas fueron de carácter secundario, tomando en cuenta documentación y bibliografía relativa a estos temas. No obstante, la gestión de la felicidad es un campo de estudio reciente, lo que limita la cantidad de información al respecto. Mediante el buscador académico Scielo se encontraron 699 artículos académicos y científicos con el término happiness, 431 con el término felicidad y únicamente 20 con los términos happiness management y 7 con los términos gestión de la felicidad. Se abordó el estudio de esta manera debido a que, en la actualidad, la Gestión de la Felicidad es un tema en el que se ha profundizado poco en el ámbito empresarial. El estudio se califica como exploratorio, con enfoque cualitativo.

\section{Discusión.}


La Gestión de la Felicidad en el entorno empresarial puede tener diversos impactos, según la naturaleza del negocio, pues esto determina el grado de participación y de interacción con el cliente que tiene el personal; no obstante, diversos estudios permiten evidenciar beneficios de esta práctica que, de manera tentativa, podrían ser aplicados en el sector turístico.

Ravina, Villena y Gutiérrez (2014) consideran que la Gestión de la Felicidad tiene efectos directos en la innovación empresarial. Según el Modelo generador de Cultura de innovación propuesto por los autores, la generación de bienestar corporativo en la fuerza de trabajo que resulta de aplicar Happiness Management eleva la creatividad del negocio, y la productividad de los recursos humanos. La productividad mejora la competitividad del negocio que hace evidente la necesidad de un comportamiento innovador, que se nutre, y a la vez fomenta, la creatividad señalada previamente. Todos estos aspectos desarrollan una cultura de innovación que se aplica mediante la gestión del conocimiento para mantener la productividad del negocio.

Para Ravina, Villena y Gutiérrez (2014) la Gestión de la Felicidad permite que las empresas sean “capaces de mejorar sus resultados innovadores y competir en el mercado no sólo por su capacidad para explotar sus recursos y sus capacidades existentes, pero también gracias a su capacidad para iniciar, renovar y desarrollar el bienestar corporativo". Sin embargo, la innovación requiere que existan condiciones factibles, tales como estímulos, capacidad y desempeño para innovar (Prajogo \& Ahmed, 2006, pág. 504). El personal que tiene un bienestar mayor se sentiría estimulado para liderar, aportar y participar en la innovación; pero la capacidad y el desempeño dependen de las condiciones técnicas y operativas de la empresa. En el sector turístico del Ecuador, una gran cantidad de servicios turísticos no se gestionan bajo un modelo empresarial, sino como negocios familiares o unipersonales, en los que es poco probable que exista una estructura, recursos y procesos para innovar. El impacto de la felicidad en la innovación empresarial del sector turístico se limitaría entonces a medianas y grandes empresas (generalmente proveedoras de alojamiento y de entretenimiento).

No obstante, la innovación es solo uno de los ámbitos en los que la Gestión de la Felicidad puede impactar para mejorar la calidad del servicio turístico y la satisfacción del turista.

Rai, Singhi, y Gupta (2012, pág. 3) concluyen que, las organizaciones que han logrado implementar la felicidad, como un objetivo para su personal, se benefician de un mayor desempeño, aumento del valor cultural y social que predomina en las relaciones entre compañeros de trabajo, el desarrollo de comunidades o grupos de trabajo cohesionados, y un alto nivel de compromiso cuando existe la posibilidad para el personal de expresarse. Las ventajas señaladas por estos autores destacan el valor del bienestar en el trabajo, para lograr mejores resultados empresariales. Se presume así, que el personal que siente felicidad manifestará una actitud más colaboradora y de equipo. Este factor es esencial en la dinámica del turismo, donde el visitante interactuar y percibe el ambiente que rodea a los equipos de trabajo que se encargan de brindarle un servicio turístico. Se destaca especialmente los servicios de alojamiento y alimentación, en los que el contacto entre el personal y el turistaes más frecuente. 
Los resultados previos conciben a la felicidad como un criterio que actúa sobre el bienestar del trabajador y que tiene múltiples efectos positivos, sin hacer distinción de la naturaleza de dicha felicidad. Por su parte Fisher (2010) reconoce múltiples constructos relacionados a la felicidad en el lugar de trabajo, mismos que agrupó en tres categorías: nivel transitorio, nivel personal y nivel de unidad; cada una generando consecuencias con diferente impacto en la organización:

Tabla 1. Constructos y Consecuencias de los niveles de felicidad identificados en el ámbito laboral según Fisher (2010)

\begin{tabular}{|c|c|c|c|}
\hline Categoría & Nivel de tránsito & Nivel personal & Nivel de unidad \\
\hline Constructos & $\begin{array}{l}\text { Satisfacción con el estado } \\
\text { del trabajo } \\
\text { Afecto momentáneo } \\
\text { Estado de flujo } \\
\text { Humor momentáneo en el } \\
\text { trabajo } \\
\text { Emoción en el trabajo } \\
\text { Estado de motivación } \\
\text { intrínseca } \\
\text { Disfrute de la tarea } \\
\text { Estado de compromiso }\end{array}$ & $\begin{array}{l}\text { Satisfacción laboral } \\
\text { Disposición para sentir } \\
\text { afecto } \\
\text { Compromiso } \\
\text { organizacional afectivo } \\
\text { Grado de implicación en } \\
\text { el trabajo } \\
\text { Humor típico en el trabajo } \\
\text { Compromiso } \\
\text { Vigor } \\
\text { Florecimiento y } \\
\text { prosperidad en el trabajo } \\
\text { Autoestima en el trabajo }\end{array}$ & $\begin{array}{l}\text { Satisfacción } \\
\text { colectiva/moral hacia } \\
\text { el trabajo } \\
\text { Tono afectivo grupal } \\
\text { Humor grupal } \\
\text { Compromiso de la } \\
\text { unidad de trabajo } \\
\text { Satisfacción grupal } \\
\text { con la tarea }\end{array}$ \\
\hline Consecuencias & $\begin{array}{l}\text { Se asocia con creatividad } \\
\text { y proactividad } \\
\text { Reduce conflictos } \\
\text { interpersonales } \\
\text { Apoya la negociación } \\
\text { colaborativa } \\
\text { soluciones } \\
\text { Mejora la satisfacción } \\
\text { laboral } \\
\text { Eleva las expectativas y la } \\
\text { motivación } \\
\text { Puede reducir el } \\
\text { desempeño al redirigir la } \\
\text { atención de la tarea hacia } \\
\text { la fuente de las emociones } \\
\text { positivas }\end{array}$ & $\begin{array}{l}\text { Reduce la intención de } \\
\text { deserción laboral, de } \\
\text { ausencia del puesto de } \\
\text { trabajo y y de } \\
\text { comportamiento contra- } \\
\text { productivo } \\
\text { Mejora el desempeño del } \\
\text { comportamiento } \\
\text { Mejora de la salud física }\end{array}$ & $\begin{array}{l}\text { Mayor satisfacción } \\
\text { en el cliente } \\
\text { Mayor percepción } \\
\text { del cliente sobre } \\
\text { calidad en el servicio } \\
\text { Mayor productividad } \\
\text { Mayor seguridad }\end{array}$ \\
\hline
\end{tabular}

Fuente: (Fisher, 2010)

La felicidad transitoria es momentánea y más allá de los efectos positivos a corto plazo, es la única que Fisher (2010) reporta, que puede tener un impacto negativo en la atención del trabajador. La felicidad a nivel personal y a nivel de unidad de trabajo tiene impacto en el sentido de pertenencia 
del trabajador hacia la empresa (que abarca también una mayor lealtad y compromiso) y en una percepción, por parte del cliente, de que el servicio es de mayor calidad.

Estos planteamientos de la felicidad como estimuladores para mejorar el rendimiento del personal y su actitud frente al cliente, puede contrastarse con los modelos de competitividad de los destinos turísticos para establecer una relación teórica entre ambos, que sustente la Gestión de la Felicidad como una práctica factible de ser implementada en el sector turístico del Ecuador.

El modelo de competitividad para destinos turísticos de Ritchie y Crouch (2003) plantea, como factores explicativos a: los recursos básicos y atractivos; los recursos y factores de apoyo; las políticas del destino, su planeación y desarrollo; los determinantes calificativos y amplificadores que influyen en la decisión del viaje; y la gestión del destino. Dentro de la gestión del destino se encuentra la gestión del talento humano, los sistemas de calidad en el servicio, y otros similares. Por su parte Jiménez y Aquino (2012, pág. 988) establece en su modelo de competitividad turístico, varios factores entre los que se halla "condiciones del destino", mismo que comprende a la gestión, a la planta turística, y a los factores de soporte, entre otros; entre los cuales el rol del personal es clave (Jiménez \& Aquino, 2012, pág. 988). Bajo estos modelos, y atendiendo a los constructos de felicidad de Fisher (2010), la Gestión de la Felicidad tendría un impacto sobre la gestión de los recursos y factores de apoyo y la gestión del destino. La competitividad de los destinos turísticos estaría beneficiada por un personal más amable, comprometido y atento al turista. No obstante, en los antecedentes revisados sobre satisfacción del turista en Ecuador, más del $80 \%$ suelen considerarse como satisfechos con el servicio, pero también es claro que también entran en consideración las expectativas previas que el turista se había formado respecto al servicio.

Los modelos de evaluación de la satisfacción toman como base a la Confirmación de Expectativas (Ruíz \& Palací, 2011), que plantea que, la satisfacción se produce cuando la experiencia vivida por el cliente supera las expectativas formadas, mientras que la insatisfacción se genera en el caso contrario. Sin embargo, muchas veces la expectativa del turista es limitada a la información que puede recopilar sobre el país a partir de publicaciones en redes sociales, páginas web, entre otras; lo que puede producir una expectativa baja ante servicios turísticos, pues el principal motivador para visitar Ecuador lo constituyen los atractivos naturales, su clima y geografía (Carvache, Carvache, Macas, \& Orden, 2018; Álava \& Bastidas, 2016; Cevallos \& Paguay, 2013). Una expectativa baja implica que el turista espere poco y se sienta satisfecho si el servicio cumple su promesa básica. Zapata (2018) describe que, el empleado de Latinoamérica que trabaja en contacto con el cliente, con mucha frecuencia suele ser de "estratos vulnerables, con escasa preparación formal y muy necesitados de trabajo. Sumado a ciertas expresiones verbales que median entre la excesiva cortesía y la sumisión, esto produce una sensación no siempre real de buen servicio". En otras palabras, un buen servicio no se produce solamente por una actitud amable o sumisa; sino que requiere la capacidad de empatía de reconocer en el turista la forma en que se siente por el trato dado, por el servicio y por la capacidad para responder a sus necesidades, más aún, de exceder sus expectativas. 
De acuerdo con Filep (2009) la felicidad del turista se define en tres dimensiones; significado, compromiso y emociones positivas que tienen lugar como parte de una experiencia memorable, segura, confortable, y que le provea una estimulación mental que resulte en interés o motivación. Bajo esta postura, la felicidad del turista puede ser impulsada desde la actitud de felicidad del personal, expresada como un estado anímico positivo, predisposición para servir, y compromiso para atender las necesidades del turista.

Sin embargo, Arias, Masía y Justo (2014) encontraron una correlación entre la felicidad del personal y positivismo de 0,828 (coeficiente de Pearson) y con satisfacción con la vida de 0,681 , pero no hallaron correlación entre felicidad y el síndrome de burnout en el trabajo, o con formas de afrontamiento (pasiva, activa, evitativa) de los problemas. Bajo estos resultados, si bien la felicidad apoya el bienestar del trabajador, no impide que se produzcan episodios de estrés, ni mejoran la capacidad del trabajador de responder ante problemas o imprevistos.

\section{Conclusiones.}

- La Gestión de la Felicidad surge como un modelo de gestión más humanista, en el que el empleado ha dejado de ser considerado solamente un recurso valorado según su desempeño, y se lo vislumbra como un ser social y espiritual, con necesidades y anhelos. El ámbito laboral constituye el lugar donde el empleado pasa cerca de la tercera parte de su tiempo, por lo que no puede asilársele en este entorno de todo aquello que le produce bienestar. La nueva concepción del Happiness Management (nueva, en comparación a otros modelos de gestión que se han mantenido vigentes por décadas), se enfoca en el empleado, considerando que su felicidad en el entorno laboral produce efectos positivos en su nivel de compromiso, lealtad a la empresa, entrega a su trabajo, y amabilidad y cordialidad con el cliente interno (compañeros de trabajo) y el cliente externo. También permite reducir el ausentismo y el abandono laboral según Frenking (2016) y promueve el éxito profesional de acuerdo con Boehm y Lyubomirsky (2008) y Rothman (2013).

- De acuerdo con Warr (2009) la felicidad y la infelicidad son conductores importantes, claves en el establecimiento y logro de objetivos, además de estar fuertemente relacionados a diversas formas de comportamiento como apoyar a colegas, ser amistosos y amables, sugerir mejoras, ser voluntarios para distintas tareas, entre otras actitudes que ayudan al rendimiento de los equipos de trabajo. En este sentido, Bono y Vey (2005) señalan que el comportamiento dentro de una organización está profundamente influenciado por las normas y reglas internas, y se incluye aquí al comportamiento emocional. De manera que la empresa es, en parte, responsable de la felicidad del personal.

- Los modelos de competitividad para destinos turísticos, así como los modelos de calidad, plantean dimensiones específicas en las cuales el rol del personal es determinante, tales como gestión del destino, capacidad de respuesta, atención al cliente, o similares. Estas dimensiones son solo una entre varios aspectos que definen la calidad en general y la experiencia del turista, sin embargo, de forma indirecta el personal comprometido y motivado puede influir de manera positiva en otros aspectos de calidad. 
- Como sugieren Acosta, Fernández y Mollón (2002) de la calidad de los recursos humanos para atender con profesionalidad, se deriva, en gran medida, la sensación de satisfacción del cliente sobre el destino y sus servicios turísticos. Alonso y Curiel (2012, pág. 182) señalan que la calidad en el servicio turístico se acoge a cinco dimensiones: tangibilidad, fiabilidad, capacidad de respuesta, seguridad y empatía. A excepción de la tangibilidad (referida a los aspectos físicos de las instalaciones, equipos y otros), las dimensiones de calidad en el servicio dependen en gran parte de la capacidad y actitud del personal. La fiabilidad se concentra en brindar el servicio prometido de la forma correcta, la capacidad de respuesta corresponde a la disposición para servir y ofrecer un servicio ágil, la seguridad se comprende como la voluntad y atención que los empleados manifiestan para trasmitir confianza en el turista y empatía para ofrecer un servicio personalizado.

- En cada una de las dimensiones mencionadas, un trabajador que percibe bienestar en su trabajo, se desempeñará de forma superior al empleado que se siente desdichado, o simplemente indiferente.

- Estrada y Ramírez (2010) mencionan que el bienestar laboral influye en el desarrollo del trabajo diario, incrementando la satisfacción y la competitividad del personal, su sentido de pertenencia, autoestima y autovaloración. Mediante la práctica del Happiness Management se fomenta el bienestar laboral y un clima positivo, lo que tiene consecuencias positivas para la organización en general. De acuerdo con Kemakorn (2011), las prácticas que más se relacionan con la felicidad en el trabajo son relaciones interpersonales, calidad de vida laboral y liderazgo; mientras Yuan-jiang, Ji y Su-yu (2009) señalan como factores de felicidad a la motivación personal, las características del trabajo, el nivel de ingresos, las relaciones interpersonales y el estrés en el trabajo, por lo que abordar estos aspectos serían un primer paso para mejorar el bienestar del ambiente de trabajo. No obstante, no puede trabajarse aspectos específicos de manera aislada, pues Grant, Christianson y Price (2007) identificaron que las prácticas de Happiness Management restringidas a dimensiones concretas, suelen mejorar un aspecto del bienestar mientras que otros disminuyen.

- Sloan (2005) sugiere que la felicidad debe fomentarse en un ambiente de equidad, en el que se promuevan relaciones interpersonales positivas, y menciona que a partir de datos de estudios se observó que las mujeres expresan ira con menor frecuencia y felicidad con mayor frecuencia que los hombres, por lo que el contar con diversidad de género ayuda a lograr armonía en el ambiente de trabajo.

- Para Beth y Gould (2000) la felicidad debe ser tratada como un objetivo de negocio, que a su vez guíe la implementación y desarrollo de un plan estratégico, debido a su impacto en el desempeño organizacional. Bajo este mismo enfoque Nogueira, Varela, Batista y Weiler (2017) plantean que la felicidad debe trabajarse como objetivo a largo plazo, y destacan la importancia de identificar niveles graduales de felicidad, y no tomar en cuenta la dicotomía de felicidad-infelicidad. Esta concepción estratégica de la felicidad invita a considerar como una inversión los gastos que se generen para mejorar el bienestar del personal, al esperar que el retorno se logre en un mayor desempeño y calidad del servicio. Para Guojuan, Shuling y Junli (2010) esto se conocería como "capital psicológico" de la empresa y abarcaría a conceptos como esperanza, optimismo, autoeficacia, resiliencia, creatividad, bienestar e inteligencia emocional, esta última, según Langhorn (2004), está relacionada con el desempeño. Por su parte Wood (2011) sugiere que el bienestar del empleado tiene como efectos: un 
aumento en el valor del trabajo, gestión participativa, retroalimentación del personal a la gerencia y apoyo motivacional.

- Los constructos señalados por Fisher (2010) para los niveles de felicidad transitoria, personal y de unidad, especifican aspectos como satisfacción, humor (dentro del margen de respeto necesario para que no afecte la actividad laboral, compromiso, implicación, sentido de pertenencia, entre otros; que resultan en una actitud proactiva hacia el cliente. La Gestión de la Felicidad influye en fiabilidad del servicio al lograr que el personal esté motivado para entregar el servicio de la mejor manera; en la capacidad de respuesta porque tendrá una mayor disposición para servir al cliente; en la seguridad porque su actitud feliz y amable dará al turista una imagen positiva y confiable; y en la empatía, porque su comportamiento le permitirá lograr un acercamiento amistoso y abierto con el cliente, facilitando la comunicación y la identificación de aquello que el turista espera o necesita.

- La Gestión de la Felicidad se presenta como una práctica administrativa que tiene un alcance amplio, pues sus acciones directas sobre el bienestar del trabajador derivan de forma indirecta en ventajas y beneficios que apoyan el logro de la calidad, sin embargo, debe tenerse en cuenta que esta no es su finalidad. Si las acciones que se apliquen para beneficio del personal son evaluadas o devengadas por el aumento del desempeño del trabajador, o de la rentabilidad del negocio, será perceptible para el trabajador, lo que afectaría se percepción sobre la organización. Para que pueda llevarse a cabo una Gestión de la Felicidad exitosa, debe partirse de objetivos claros dirigidos a reconocer en el trabajador sus necesidades y anhelos, y dotarle de aquello que esté dentro de las posibilidades de la organización. Los efectos sobre el desempeño, satisfacción del cliente, calidad, entre otros; deben manifestarse como efectos del estado del trabajador, de modo que sean sus decisiones y actitudes las que se encaminen al beneficio empresarial.

- Actualmente existen grupos de investigación en la Universidad Politécnica Salesiana del Ecuador, que están abordando el carácter multidisciplinar de la felicidad y happiness management, lo que demuestra la relevancia que está despertando este tema en ámbitos tan diversos como la psicología, la administración, el marketing, recursos humanos, entre otros. Los estudios que se desarrollen en el ámbito de la Gestión de la Felicidad, deben adoptar una postura, tanto humana y social, como técnica y laboral. El impacto de esta práctica puede diferir de empleado en empleado, pero en conjunto, al trabajar sobre la felicidad a nivel de unidad (Fisher, 2010), los beneficios en la organización deberían ser medibles; no obstante, se desconoce actualmente en que aspectos es donde presenta un mayor impacto. La evidencia existente al momento, permite suponer que, en empresas de servicios, donde el personal tiene un contacto frecuente con el cliente, los efectos del Happiness Management influyen en la calidad de la atención, por lo que se percibe, además, la oportunidad de que surjan futuras investigaciones que relacionen esta práctica con los beneficios que puede tener en las empresas de producción, donde el contacto con el cliente es reducido o nulo. En resumen, se sugieren como líneas de investigación: medición de la felicidad a nivel grupal y de individuo como parte de una organización, impacto cuantitativo de la felicidad en la calidad de atención en empresas de servicio e impacto de la felicidad en los resultados de productividad de empresas de producción. 


\section{Referencias Bibliográficas.}

Acosta, A., Fernández, N., y Mollón, M. (2002). Recursos humanos en empresas de turismo y hotelería. Madrid, España: Pearson Educación.

Álava, L., y Bastidas, J. (2016). Estudio de la Calidad del Servicio y Satisfacción del cliente de las Agencias de Viaje en Puerto Ayora, Isla Santa Cruz. Trabajo de Titulación de Licenciatura, Universidad Central del Ecuador, Carrera de Turismo Ecológico, Puertoy Ayora, Islas Galápagos.

Alonso, A., y Curiel, J. (2012). Turiso y Relaciones Internacionales. Aspectos sociales, culturales, económicos y ambientales. España: Universidad Rey Juan Carlos.

Aranda, E. (2016). La gerencia de la felicidad: un nuevo modelo para la gestión de las organizaciones. Universidad Militar Nueva Granada, Bogotá, Colombia.

Arias, W., Masía, A., y Justo, O. (2014). Felicidad, Síndrome ed Burnout y estilos ed afrontamiento en una empresa privada. Revista de la Facultad de Psicología y Humanidades, 22(1), 7588 .

Ayu, Y., y Lyana, F. (2018). A conceptual paper on the Theory of HCappiness in Neighbourhood. Asian Journal of Behavioural Studies, 3(12), 1-12. doi:10.21834/ajbes.v3i12.117

Beth, H., y Gould, K. (2000). Strategic Planning for Employee Happiness: A Business Goal for Human Service Organizations [Planificación estratégica para la felicidad del pertsonal: un objetivo de negocios para organizaciones de servicios humanos]. American Journal on Mental Retardation, 105(5), 377-386.

Boehm, J., y Lyubomirsky, S. (2008). Does Happiness Promote Career Success? [¿La felicidad promueve el éxito profesional?]. Journal of Career Assessment, 16(1), 101-116. doi: $10.1177 / 1069072707308140$

Bono, J., y Vey, M. (2005). Toward Understanding Emotional Management at Work: A Quantitative Review of Emotional Labor Research. En H. Charmine, N. Ashkanasy, y W. Zerbe, Emotions in Organizational Behavior. New York, USA: Psychology Press.

Carvache, M., Carvache, W., Macas, C., y Orden, M. (2018). Motivaciones, Valoración y Satisfacción del Turista en un destino de Sol y Playa de Ecuador. Revista Espacios, 39(13).

Carvache, W., Torres, M., y Carvache, M. (2017). Análisis del perfil y satisfacción del turista que visita Montañita-Ecuador. Revista Cuadernos de Turismo(39), 113-129.

Cevallos, F., y Paguay, J. (2013). Análisis del Perfil Socioeconómico de los Turistas que visitan las Islas Galápagos y medición de su nivel de Satisfacción de su visita al destino. Revista Res Non Verba, 60-74.

Del Pozo, J. (2015). Procesos de gestión de calidad de hostelería y turismo. Málaga, España: IC Editorial. 
Edwards, C. (2009). The pursuit of happiness: human resource management [La búsqueda de la felicidad: Gestión de Recursos Humanos]. Engineering y Technology, 4(4), 76-79. doi:10.1049/et.2009.0419

Encalada, E. (05 de febrero de 2019). Las inversiones en turismo serán las más bajas en ocho años. Diario El Comercio.

Espín, T. (2014). Análisis de Satisfacción del Turista respecto a los servicios de alojamiento urbano en Baños de Agua Santa. Tesis de Ingeniería, Universidad Regional Autónoma de los Andes, Facultad de Dirección de Empresas, Ambato, Ecuador.

Estrada, Y., y Ramírez, M. (2010). El Bienestar Laboral y su Incidencia en la Gestión Exitosa de las Empresas en el Turismo. TuryDes Revista de investigación en Turismo y Desarrollo Local, 3(8).

Filep, S. (2009). Tourists' happiness through the lens of positive psychology [Felicidad del turista a través del lente de la psicología positiva]. Tesis, James Cook University, Australia.

Fisher, C. (2010). Happiness at Work [Felicidad en el trabajo]. International Journal of management Reviews, 12, 384-412. doi:10.1111/j.1468-2370.2009.00270.x

Frenking, S. (2016). Feel Good Management as valuable tool to shape workplace culture and drive employee happiness [Gestión del bienestar como herramienta valiosa para formar la cultura laboral y conducir la felicidad del empleado]. Strategic HR Review, 15(1), 14-19. doi:10.1108/SHR-11-2015-0091

Grant, A., Christianson, M., y Price, R. (2007). Happiness, Health, or Relationships? Managerial Practices and Employee Well-Being Tradeoffs []. ¿Felicidad, salud o relaciones? Prácticas Gerenciales y Compromisos de Bienestar de los Empleados, 21(3), 51-63. doi:10.5465/amp.2007.26421238

Griffiths, J., Johnson, F., y Hartley, R. (2007). User satisfaction as a measure of system performance. Journal of Librarianship and Information Science, 39(2), 142-152.

Guojuan, Z., Shuling, W., y Junli, Z. (2010). Research on the Happiness Management Model from de Perspective of Psychological Capital [Investigación del Modelo de Gestión de la Felicidad desde la perspectiva de Capital Psicológico]. Proceedings of the 7th International Conference on Innovation y Management, 1733-1737.

Hartini, S., Khalil, M., y Zakaria, A. (2017). The mediatinf effect of happiness on the relationship between work-life balance and life satisfaction. Social and Management Research Journal, 14(2), 33-54.

Jiménez, P., y Aquino, F. (2012). Propuesta de un modelo de competitividad para destinos turísticos. Revista Estudios y Perspectivas en Turismo, 21(4), 977-995. 
Kemakorn, O. (2011). Happiness at Work of employees in Small and Medium-sized Enterprises, Thailand [Felicidad en el trabajo de empleados en pequeñas y medianas empresas]. Procedia - Social and Behavioral Sciences, 25, 189-200. doi:10.1016/j.sbspro.2011.10.540

Langhorn, S. (2004). How emotional intelligence can improve management performance [Cómo la inteligencia emocional pueude mejorar el desempeño gerencial]. INTERNATIONAL JOURNAL OF CONTEMPORARY HOSPITALITY MANAGEMENT, 16(4), 220-230. doi:10.1108/09596110410537379

Nogueira, J., Varela, C., Batista, M., y Weiler, M. (2017). Creativity and innovation for corporate happiness management [Creatividad e innovación para la gestión de feicidad corporativa]. Brazilian Journal of Science and Technology, 4(1). doi:10.1186/s40552-017-0038-7

Organización Mundial del Turismo. (2018). Panorama OMT del turismo internacional. Obtenido de World Tourism Organization: https://www.eunwto.org/doi/pdf/10.18111/9789284419890

Parasuraman, A., Zeithaml, V., y Berry, L. (1985). A conceptual model of Service Quality and its Implications for Future Research [Un modelo conceptual de Calidad en el servicio y sus implicaciones para futuras investigaciones]. The Journal of Marketing [La revista de Marketing], 49(4), 41-50.

Prajogo, D., y Ahmed, P. (2006). Relationships between innovation stimulus, innovation capacity, and innovation performance. R y D Management, 36(5), 499-515.

Rai, V., Singhi, M., y Gupta, R. (2012). From performance managemente system to happiness management system [De un sistema de gestión del desempeño a un sistema de gestión de la felicidad]. Dream, Discove, Dare, innovations in the Global Village: Role of International HRM. India.

Ravina, R., Villena, F., y Gutiérrez, G. (2014). Una aproximación teórica para mejorar los resultados de innovación en las empresas desde la perspectiva del "Happiness Management". Retos. Revista de Ciencias de la Administración y Economía, 7(14). doi:10.17163/ret.n14.2017.06

Ritchie, B., y Crouch, G. (2003). The Competitive Destination: A Sustainable Tourism Perspective [Destinos Competitivos: Una perspectiva de turismo sustentable]. Reino Unido: CABI Publishing.

Romero, L., Castillo, B., y Ravina, R. (2019). Comunicación para la efectividad laboral y el happiness management: Revisión crítico-analítica de la literatura. Revista Mundo Indess, 17-21.

Rothmann, S. (2013). From Happiness to Flourishing at Work: A Southern African Perspective [De la felicidad al florecimiento en el trabajo: una perspectiva surafricana]. En M. Wissing, 
Well-Being Research in South Africa. Cross-Cultural Advancements in Positive Psychology (págs. 123-151). Springer Science+Business Media.

Ruíz, M., y Palací, F. (2011). Variables cognitivas y psicología del consumidor. El modelo de la confirmación de expectativas en la actualidad. Boletín de Psicología, 103, 61-73.

Sloan, M. (2005). "There is no happiness at work!": emotion management, inauthenticity, and psychological distress in the workplace [“ $i N o$ hay felicidad en el trabajo!”: Manejo de las emociones, falta de autenticidad y angustia psicológica en el lugar de trabajo]. Tesis doctoral, Vanderbilt University.

Turienzo, R. (2016). El pequeño libro de la motivación. España: Grupo Planeta.

Varenius, N. (2016). Descripción del grado de satisfacción del turista que visita la Isla Santay. Trabajo de Investigación para ingeniería, Universidad Casa Grande, Facultad de Administración y Ciencias Políticas, Guayaquil, Ecuador.

Warr, P. (2009). Environmental "Vitamins", Personal Judgments, Work Values, and Happiness ["Vitaminas" ambientales, juicios personales, valores laborales y felicidad]. The Oxford Handbook of Organizational Well Being. doi:10.1093/oxfordhb/9780199211913.003.0004

Wesarat, P.-o., Yazam, M., y Abdul, A. (2015). A Conceptual Framework of Happiness at the Workplace. Asian Social Science, 11(2), 78-88. doi:10.5539/ass.v11n2p78

Wood, S. (2011). High involvement management, high-performance work systems and wellbeing. The International Journal of Human Resource Management, 22(7), 1586-1610. doi:10.1080/09585192.2011.561967

World Tourism Organization. (2019). International Tourist Arrivals Reach 1.4 billion Two Years Ahead of Forecasts. Obtenido de World Tourism Organization: http://www2.unwto.org/press-release/2019-01-21/international-tourist-arrivals-reach-14billion-two-years-ahead-forecasts

Xinggui, Z., Yang, G., Ming, D., y Zhanpeng, Z. (2018). Chines Job Well-Being: its concept and scale developing. International Journal of Business Anthropology, 8(1), 25-38. doi:10.33423/ijba.v8i1.1100

Yuan-jiang, M., Ji, F., y Su-yu, B. (2009). An Overview of Happiness in the Workplace [Una visión general de la felicidad en el lugar de trabajo]. Economic Management Journal, 10, $179-186$.

Zapata, B. (26 de abril de 2018). Atención al cliente con falencias en Ecuador. Diario El Universo.

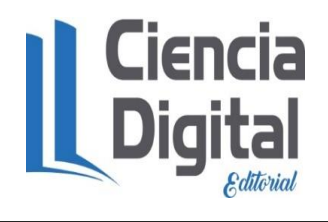




\section{PARA CITAR EL ARTÍCULO INDEXADO.}

Paladines, G. V., Suárez Velasco, J. E., \& Capa Paladines, S. F. (2020). Gestión de la felicidad y satisfacción del turista en Ecuador. Conciencia Digital,3(4), 122-138. https://doi.org/10.33262/concienciadigital.v3i4.1432

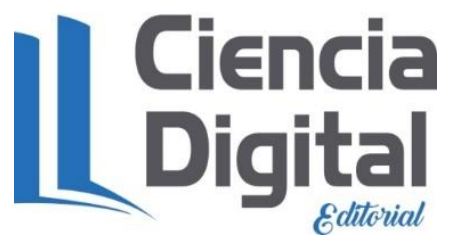

El artículo que se publica es de exclusiva responsabilidad de los autores y no necesariamente reflejan el pensamiento de la Revista Conciencia Digital.

El artículo queda en propiedad de la revista y, por tanto, su publicación parcial y/o total en otro medio tiene que ser autorizado por el director de la Revista Conciencia Digital.

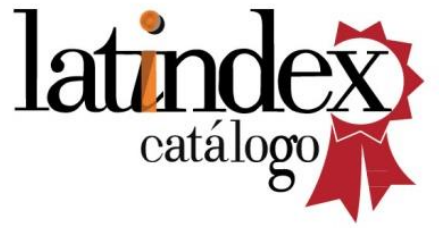

\section{Conciencia}

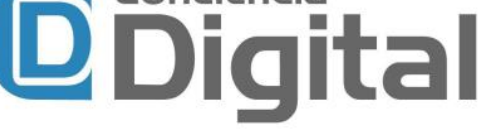

